UNUSUAL COMPLICATION IN A DIFFICULT TO DIAGNOSE CASE OF CNS TUBERCULOSIS

Dr. Suhas V. Prabhu, Consultant Pediatrician, P.D. Hinduja hospital, Mumbai, INDIA

\section{Case History:}

13 month old boy admitted with h/o Fever and cough for 15 days and convulsions and left sided weakness for 3 days. Normal history and development till present illness which was of insidious onset, fever became high later. Cough dry, spasmodic, gradually worsening in severity and intensity, almost whoopy.

\section{On Examination:}

Weight: $8.5 \mathrm{Kg}$, Length: $79 \mathrm{~cm}$, Head: $44 \mathrm{~cm}$. Afebrile, HR 140/m, RR $45 / \mathrm{m} \mathrm{BP} 114 / 68$. Pallort, no rash, icterus, clubbing or lymph node enlargement. RS: Bilateral wheeze and some creps +. Mild hepatosplenomegaly. CNS: GCS 9/15, Pupils CCERL, Cr. N. - Normal. Left side: Mild hemiparesis, hypotonia, hyper-reflexia, plantar extensor. Bilateral fine tremors.

\section{Investigations:}

Normal biochemistry except mildly elevated AST and ALT 68 \& $70 \mathrm{U} / \mathrm{L}$. Blood counts are as shown in the table.

\begin{tabular}{|c|c|c|c|c|c|}
\hline Date & $11 / 5$ & $16 / 5$ & $21 / 5$ & $24 / 5$ & $29 / 5$ \\
\hline $\mathrm{Hb}(\mathrm{gm} / \mathrm{dl})$ & 7.8 & 7.3 & 6.8 & 10.2 & 8.9 \\
\hline Total $\mathrm{WBC} / \mathrm{\mu L}$ & 48,200 & 68,800 & 76,500 & 50,750 & 15,900 \\
\hline Polys \% & 36 & 50 & 38 & 45 & 49 \\
\hline Platelets/ $\mathrm{\mu L}$ & 394,000 & 533,000 & 399,000 & 545,000 & 506,000 \\
\hline
\end{tabular}

CRP - negative. X-ray chest: bilateral infiltrates, no hilar or mediastinal lymphadenopathy. CSF: Proteins $81 \mathrm{mg} / \mathrm{dl}$, Glucose $94 \mathrm{mg} / \mathrm{dl}$, WBC 114 cells/ cu. mm. P 45\%, L 55\%. No organisms seen. PCR for $M$ tb negative. Gastric lavage for $M$. tb - negative. Blood and urine cultures - no growth. Mantoux test - negative. Anti-Mycoplasma IgM negative. Serum iron: 8 mg\%, TIBC 241 mg\%, TS 3.3\%. Ferritin: 81.2 $\mathrm{mg} \%$

MRI brain - T2 weighted images:

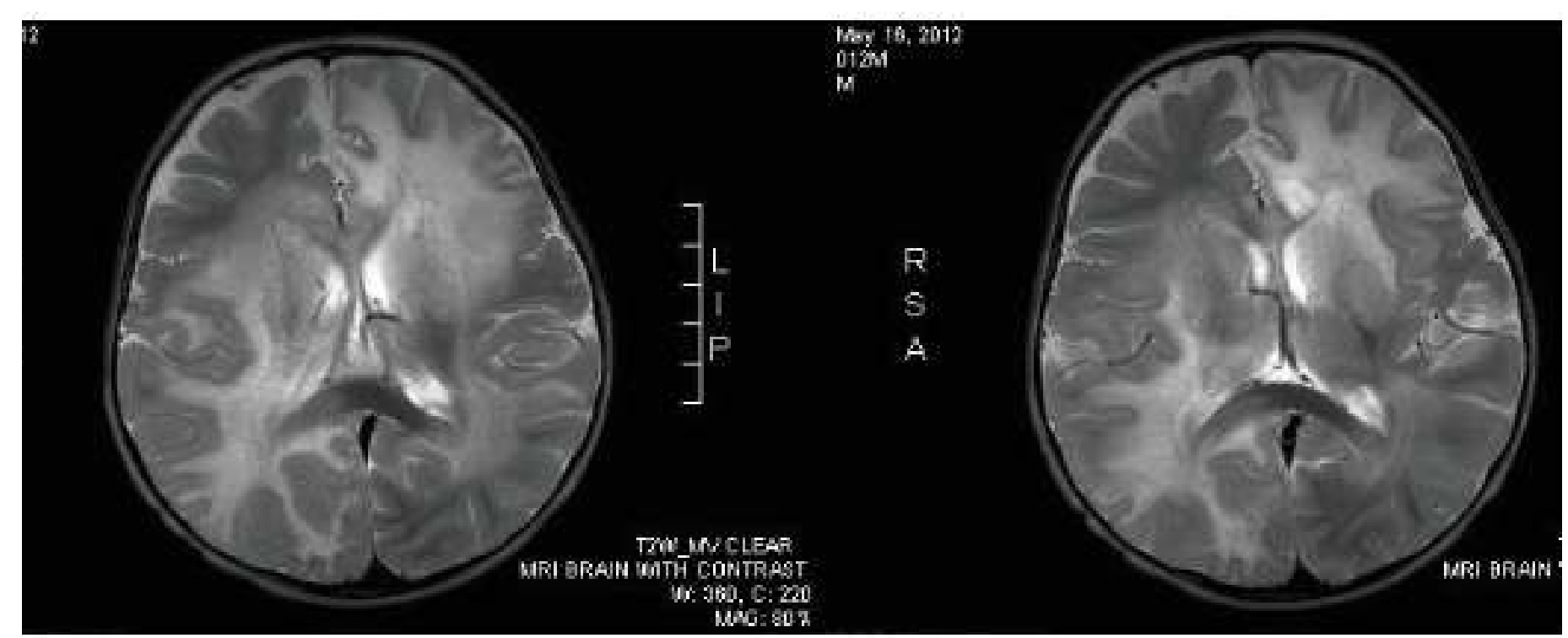

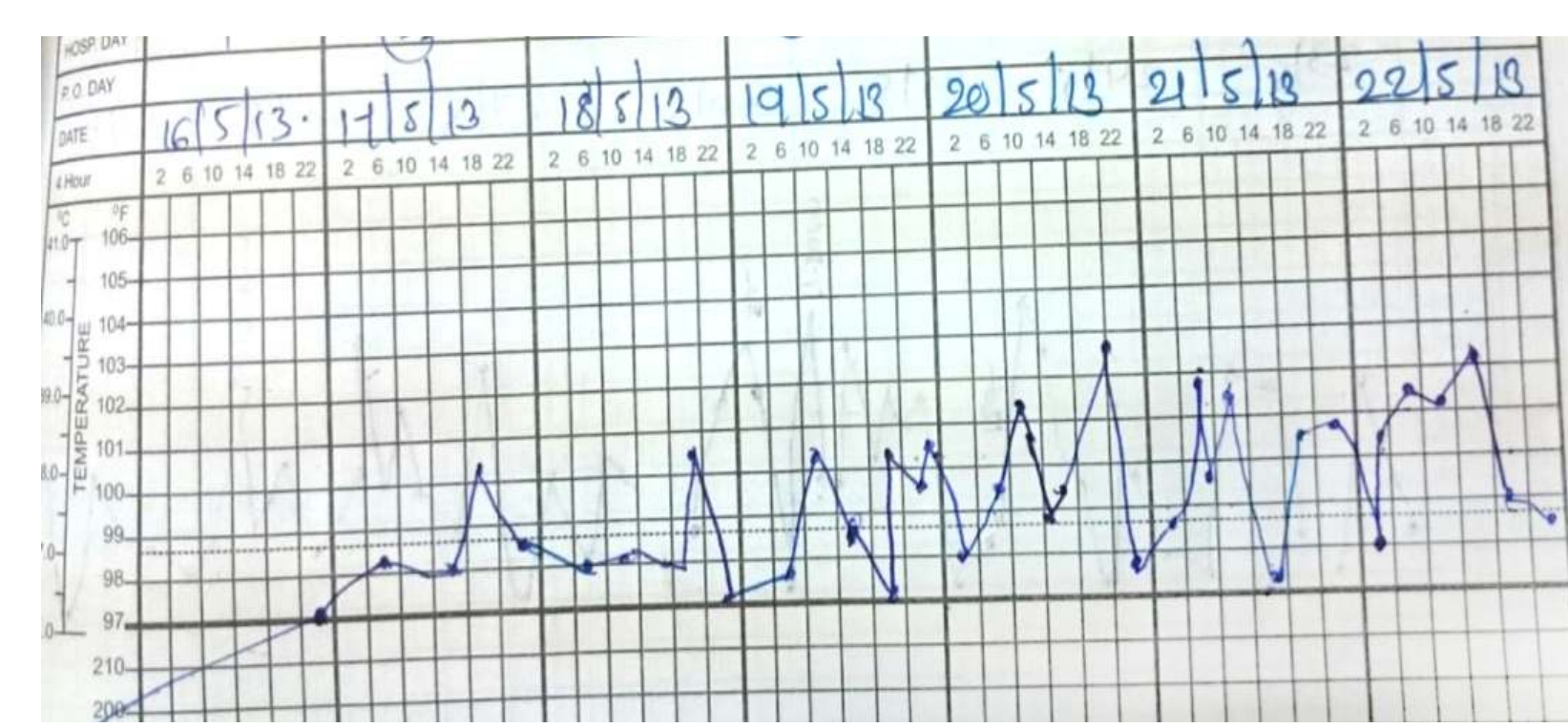

4 drug AKT - HRZE along with steroids started empirically on $22 / 5$. Sensorium improved, recognizing mother, able to feed orally, hemiparesis unaltered. Cough subsided gradually, ches cleared, confirmed on X-ray chest. But continued to run high fever. Repeat cultures (blood, urine) no growth.

Then threw a series of convulsions with worsening of sensorium and regression. Fresh set of investigations done.. Repeat CSF: Proteins 86 mg\%, Glu 88 mg\% WBC 680 (P63,L15,M22). No AFB seen, culture no growth. TB PCR -ve, HSV PCR -ve. Cryptococcus antigen -ve. Viral studies (PCR panel) - ve. IgG $5.8(\mathrm{~N})$; Lactate - $11 \mathrm{mg} \%$ (normal). HIV Elisa - negative. BAL - negative for AFB (WBC $600 \mathrm{~N}-11 \%$ )

$M R I$ repeated: Mild improvement in lesions.

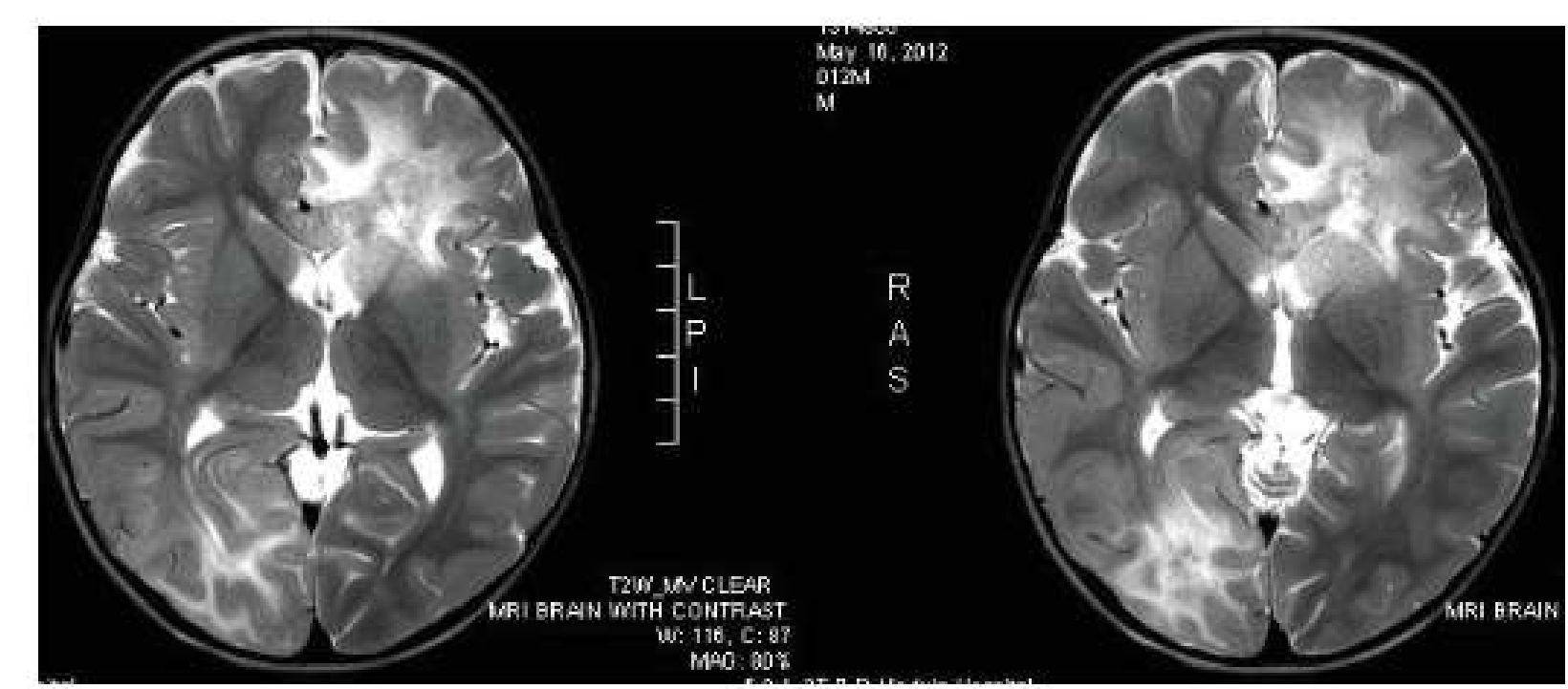

But granuloma like lesions now seen:

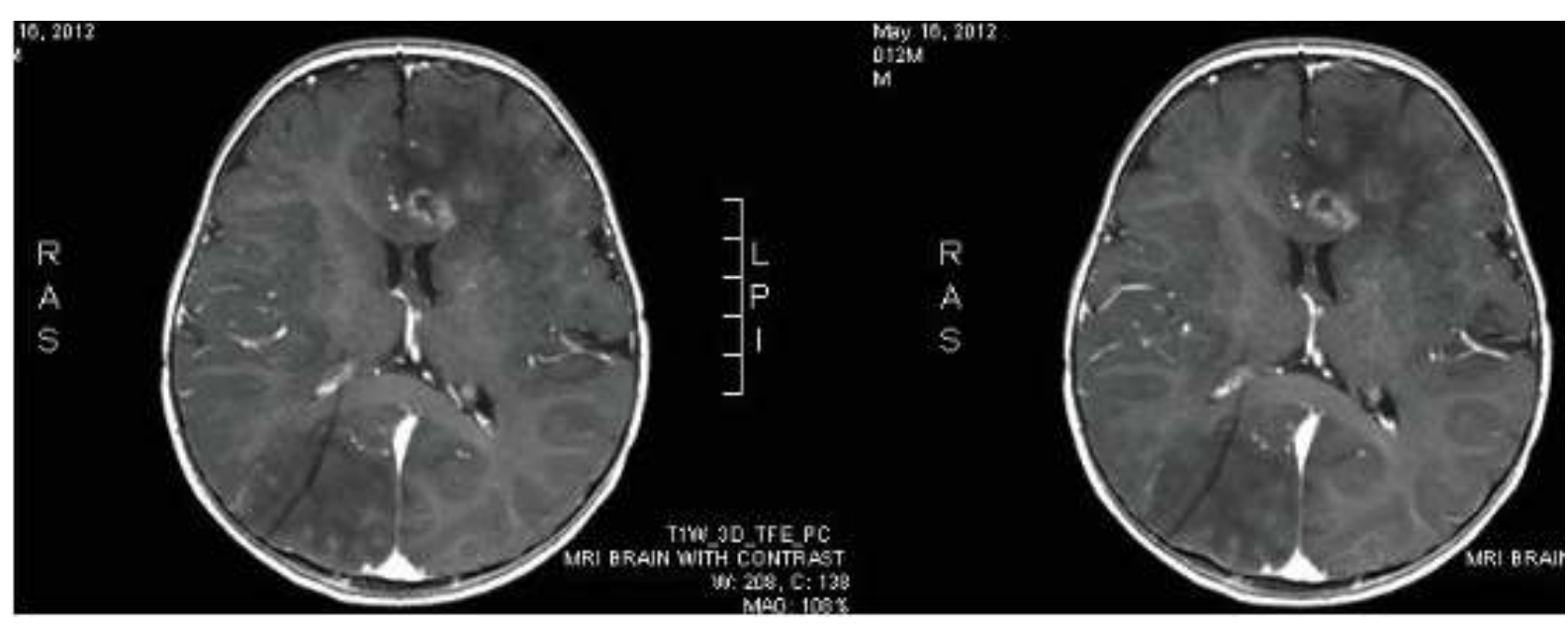

And high fever continued with poor neurological improvement.

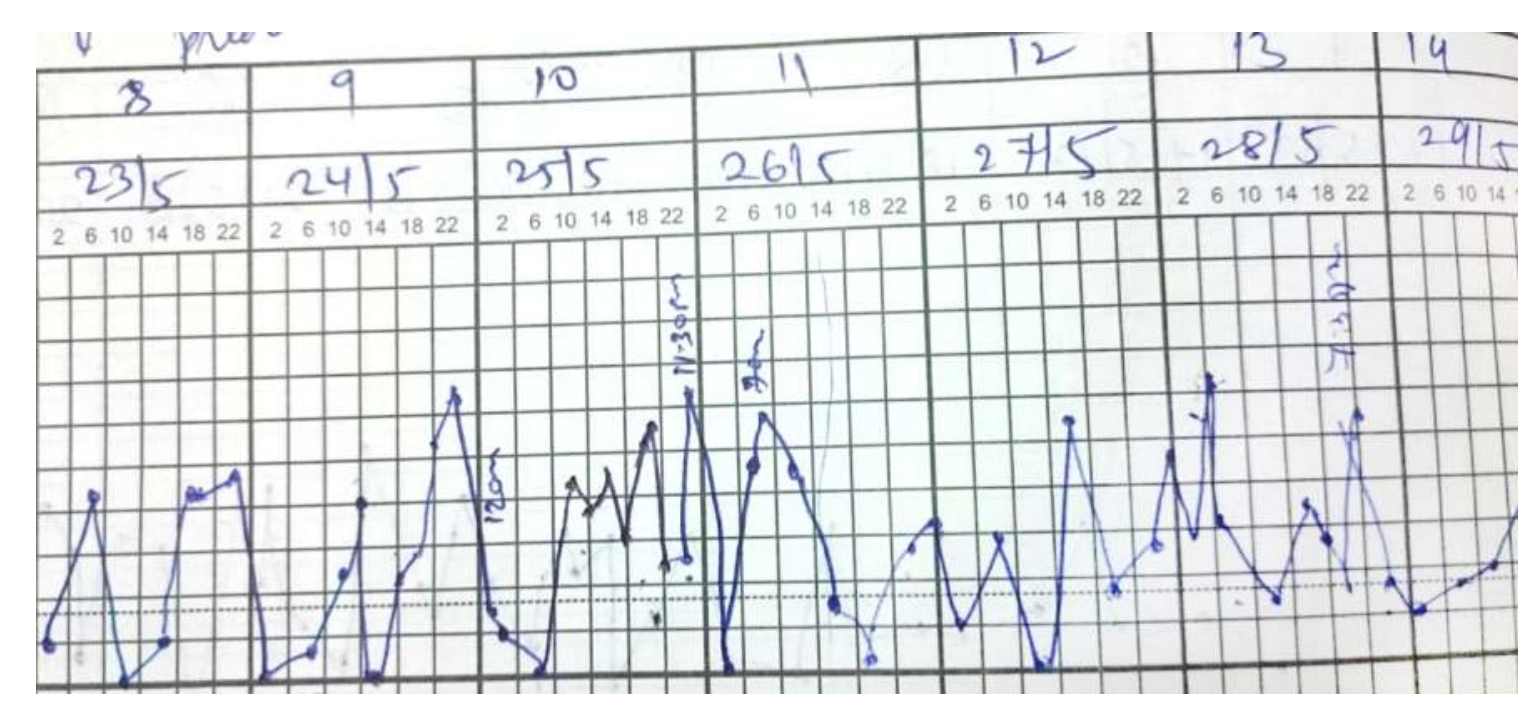

Stereotactic brain biopsy done on $31 / 5$. White matter showed increased cellularity. Scattered reactive glial cells, large number of CD68 +ve histiocytes, no caseation, no granulomas. No organisms seen. Z-N stain - negative for AFB. Conclusion: Florid inflammatory pathology, suggestive of Haemophagocytic Lymphohistiocytosis (HLH)!

No evidence of HLH elsewhere. No cytopenia

hypofibrinogenemia - $166 \mathrm{mg} \%$, hypertriglyceridemia $140 \mathrm{mg} \%$. Bone marrow aspiration: no evidence of haemophagocytosis. Perforin level - 94\% (Normal). But AKT omitted and started on HLH protocol with pulse methylprednisolone and Etoposide/Cyclosporin.

Only mild clinical improvement seen. on $16 / 6$, brain biopsy AFB culture report growing M. tuberculosis! AKT re-started. Patient gradually improved and discharged. 6 months later, had improved enough to walk unaided and started babbling. Later developed secondary epilepsy, difficult to control and ended up retarded.

Final Diagnosis: CNS tuberculosis with localized Haemophagocytic Lymphohistiocytosis.

\section{Take Home Messages:}

- TBM is still a common and dangerous disease with myriad forms of presentation; so we MUST think of it

- Difficult to make a diagnosis, so attempt bacteriological

diagnosis always! That is the only foolproof diagnosis. -TB can induce HLH (confined to local area)

- "Cure" from severe forms of TBM can still leave a devastated child and family, so early diagnosis and prompt treatment is vital.

Acknowledge the assistance of Dr Vrajesh Udan (Pediatric Neurologist), Dr Camilla Rodrigues (Microbiologist) \& Dr Milind Sankhe (Neurosurgeon) 